Para uso de acordo com a Lei do Direito Autoral 9.610/98. 


\section{QUASI PARTICLE ENERGY OF 4f-STATES IN THE RAMIREZ-FALICOV-KIMBALL (RFK) MODEL: MEMORY FUNCTION FORMALISM}
I. C. da Cunha Lima $\left({ }^{1}\right)$, C. E. Leal $\left({ }^{1}\right)$, E. A. de Andrada e Silva $\left({ }^{1}\right)$ and A. Troper $\left({ }^{2}\right)$
$\left.{ }^{1}\right)$ Instituto de Pesquisas Espaciais, av. dos Astronautas, 1758, 12.200 São Jose dos Campos, SP, Brazil $\left(^{2}\right)$ Centro Brasileiro de Pesquisas Fisicas - CBPF, Rua Dr. Xavier Sigaud, 150, 22290 Rio de Janeiro, RJ,
Brazil

Abstract. - A new formalism is developed, based on the memory function approach, to treat many particle systems. The formalism is applied to the Ramirez-Falicov-Kimball (RFK) Hamiltonian, suitable to describe photoemission spectra in many light rare earth intermetallics. We obtain a quasi particle 4 f-energy in the weak correlation regime and we discuss the bimodal structure of the f-f propagator in this regime comparing with the Hubbard-type structure in the strong correlation regime.

It is well known that many experiments concerning the photo-emission of 4 -electrons in light rareearth elements, e.g., Ce, show a double peak structure: one localized at the Fermi level and another approximately $2.5 \mathrm{eV}$ below it.

Parks et al. [1] and Wieliczka et al. [2] have shown that this bimodal structure of the $4 \mathrm{f}$-spectra occurs in many other metallic systems containing light rare earths such as $\mathrm{Pr}$ and $\mathrm{Nd}$.

Many works $[3,4,5]$ have been proposed in order to explain the $4 \mathrm{f}$-double structure, based, for example, on the rare earth magnetic properties [3] or on screening effects $[4,5]$. Nunez-Regueiro and Avignon [6] have calculated the $4 \mathrm{f}$-spectral density, based on the FalicovKimball model, adopting Hubbard's "resonance broadening approximation". This strong correlation regime approximation, yields one or two peaks depending on the ratio between the Coulomb correlation $U$ between the f-localized states and the d-itinerant states and the $\mathrm{d}$-bandwidth $\Delta$. Moreover, $\mathrm{f}$-d hybridization plays no significant role in the broadening of the two peaks.

In this work, adopting the Ramirez-Falicov-Kimball (RFK) Hamiltonian, we calculate the f-f Green's function in the weak correlation regime, i.e., $U / W<1$. We develop here a Memory Function matrix formalism, which enables us to describe the weak correlation regime beyond the usual Hartree-Fock approximation.

For the sake of simplicity, we discuss here only the RFK Hamiltonian in the one-impurity case:

515

6) 277 .

1. Rojo, A. and 1941.

17 (1974) 31.

. and Iglesias, rmun.

(1960) 320.

L1037.

Rev. $B \mathbf{2 0}$
$H=\sum_{\sigma} \varepsilon_{0} f_{0 \sigma}^{+} f_{0 \sigma}+\sum_{\mathbf{k}_{\sigma}} \varepsilon_{\mathbf{k}} d_{\mathbf{k}_{\sigma}}^{+} d_{\mathbf{k}_{\sigma}}+$

$+\sum_{\mathbf{k}_{\sigma}} V\left(F_{0 \sigma}^{+} d_{\mathbf{k}_{\sigma}}+d_{\mathbf{k}_{\sigma}}^{+} f_{0 \sigma}\right)+\sum_{\sigma \sigma^{\prime}} U n_{0 \sigma}^{\mathrm{d}} n_{0 \sigma^{\prime}}^{\mathrm{f}} ;$

$n_{0 \sigma}^{\alpha}=\alpha_{0 \sigma}^{+} \alpha_{0 \sigma^{\prime}}$;

$\sum_{\sigma} n_{0 \sigma}^{\alpha}=n_{0}^{\alpha}, \quad(\alpha=f$ or $\mathrm{d})$.

The local $\mathrm{f}-\mathrm{f}$ Green function is given by

$$
G_{00 \sigma}^{\mathrm{ff}}(t)=i \theta(t)\left\langle\left[f_{0 \sigma}, f_{0 \sigma}^{+}(t)\right]_{+}\right\rangle .
$$

Now we introduce the self-consistent many body theory developed by Fedro and Wilson [7], Kishore [8] and Chao et al. [9]. Let us consider two sets of Heisenberg fermion operators $A_{\alpha}$ and $B_{\beta}$ forming a complate space:

$$
\begin{aligned}
& \left\{A_{\alpha}\right\}=\left\{f_{0 \sigma}, d_{\mathbf{k}_{\sigma}}\right\} \\
& \left\{B_{\beta}\right\}=\left\{f_{0 \sigma}^{+}, d_{\mathbf{k}_{\sigma}}^{+}\right\}
\end{aligned}
$$

and a projection operator $P$ defined as

$$
P \Psi=\sum_{j} P_{j} \Psi=\sum_{j} B_{j} \frac{\left\langle\left[A_{j}, \Psi\right]_{+}\right\rangle}{\left\langle\left[A_{j}, B_{j}\right]_{+}\right\rangle} .
$$

Using the sets given by equation (3), we have:

$$
P \Psi=f_{0 \sigma}^{+}\left\langle\left|f_{0 \sigma}, \Psi\right|_{+}\right\rangle+\sum_{\mathbf{k}} d_{\mathbf{k}_{\sigma}}^{+}\left\langle\left[d_{\mathbf{k}_{\sigma}}, \Psi\right]_{+}\right\rangle \text {. }
$$

An equation of motion for the matrix $\bar{G}(w)$ :

can be worked out:

$$
G_{\alpha \beta}(t)=i \theta(t)\left\langle\left[A_{\alpha}, B_{\beta}(t)\right]_{+}\right\rangle
$$

$$
\tilde{G}(w)=[x \tilde{I}-\tilde{\Omega}-\tilde{\gamma}(w)]^{-1} \tilde{\chi}
$$

where

$$
\begin{aligned}
\Omega_{\alpha \beta} & =\frac{\left\langle\left[A_{\alpha} L B_{\beta}\right]_{+}\right\rangle}{\left\langle\left[A_{\alpha}, B_{\alpha}\right]_{+}\right\rangle} \\
\chi_{\alpha \beta} & =\left\langle\left[A_{\alpha}, B_{\beta}\right]_{+}\right\rangle \delta_{\alpha \beta}
\end{aligned}
$$

and

$$
\gamma_{\alpha \beta}(w)=\left\langle\left[A_{\alpha}, L \frac{1}{w-(1-P) L}(1-P) L B_{\beta}\right]_{+}\right\rangle \text {, }
$$

$L$ being the Liouvillean operators: $L \Psi \equiv[H, \Psi]$.

If we identify our first matrix element with the fstate, we have:

$$
G_{00 \sigma}^{\mathrm{ff}}(w)=[w \tilde{I}-\tilde{\Omega}-\tilde{\gamma}(w)]_{11}^{-1} \chi_{11} .
$$

Equation (11) can be solved in several levels of approximations for the matrix $\tilde{\gamma}(w)$. In the lowest level of approximation we use the linearized $\mathrm{f}-\mathrm{d}$ Coulomb term in the Hamiltonian. Then we find: $\tilde{\gamma}(w)=0$. The f-f propagator becomes:

$$
G_{00 \sigma}^{\mathrm{ff}}(w)=\frac{1}{w-\varepsilon_{0}^{f}-U\left\langle n_{0}^{\mathrm{d}}\right\rangle-V^{2} F(w)}
$$

where

$$
F(w)=\sum_{\mathbf{k}} \frac{1}{w-\varepsilon_{\mathbf{k}}-U\left\langle n_{0}^{\mathrm{f}}\right\rangle} .
$$

and we recover the Hartree-Fock approximation. 
In the next step, we use a recursion formula for the self-energy $\gamma(w)[9,10]$.

The hierarchy of the Green's function is truncated by approximating conveniently the self-energy $\gamma^{\mathrm{ff}}(n+1: w)$. Thus, in the first order approximation, we linearize the Hamiltonian for $\gamma^{\mathrm{ff}}(2: w)$, which will give us again $\gamma^{\mathrm{ff}}(2: w)=0$. Then we obtain from the recursion formula:

$$
\gamma^{\mathrm{ff}}(1: w)=\frac{U^{2}\left\langle n_{0}^{\mathrm{d}}\right\rangle\left(1-\left\langle n_{0}^{\mathrm{d}}\right\rangle\right)}{w+\varepsilon_{0}+U\left\langle n_{0}^{\mathrm{d}}\right\rangle} .
$$

$G_{00 \sigma}^{\mathrm{ff}}$ exhibits a bimodal structure in the weak correlation regime. This bimodal structure however is quite different from the Hubbard-type two-peak structure [6] which is peculiar to a strong correlation regime. The two resonant f-energies are:

$$
E_{ \pm}=\frac{V^{2} F(w)}{2} \pm \frac{1}{2} \sqrt{\left[2 \varepsilon_{0}+2 U\left\langle n_{0}^{\mathrm{d}}\right\rangle+V^{2} F(w)\right]^{2}+4 U^{2}\left\langle n_{0}^{\mathrm{d}}\right\rangle\left(1-\left\langle n_{0}^{\mathrm{d}}\right\rangle\right)}
$$

The f-f propagator, exhibiting a n-modal structure is obtained by linearizing again the Coulomb interaction contribution for higher $\gamma^{\mathrm{ff}}(n+1: w)$ terms in the recursion formula. As an illustration of this peculiar feature, we perform the calculation up to a higher level of approximation, truncating the expansion terms in $\gamma^{\mathrm{ff}}(3: w)$, giving rise to terms in $U^{3}$. Then, we have:

$$
\gamma^{\mathrm{ff}}(w)=\frac{\left(w\left\langle\left[f_{0 \sigma}, L(1-P) L f_{0 \sigma}^{+}\right]_{+}\right\rangle+\left\langle\left[f_{0 \sigma}, L^{2}(1-P) L f_{0 \sigma}\right]_{+}\right\rangle\right)}{w^{2}+w\left\langle\left[f_{0 \sigma}, L f_{0 \sigma}^{+}\right]_{+}\right\rangle+\left\langle\left[f_{o \sigma}, L^{2} f_{0 \sigma}^{+}\right]_{+}\right\rangle}
$$

and after some algebra we obtain:

$$
\gamma^{\mathrm{ff}}(w)=\frac{w U^{2}\left\langle n_{0}^{\mathrm{d}}\right\rangle\left(1-\left\langle n_{0}^{\mathrm{d}}\right\rangle\right)+U^{2}\left\langle n_{0}^{\mathrm{d}}\right\rangle\left(1-\left\langle n_{0}^{\mathrm{d}}\right\rangle\right)\left(2 \varepsilon_{0}+U\right)+V^{2} U\left(\left\langle n_{0}^{\mathrm{f}}\right\rangle-\left\langle n_{0}^{\mathrm{d}}\right\rangle\right)}{w^{2}+w\left(\varepsilon_{0}+U\left\langle n_{0}^{\mathrm{d}}\right\rangle\right)+\left(\varepsilon_{0}^{2}+2 \varepsilon_{0} U\left\langle n_{0}^{\mathrm{d}}\right\rangle+U^{2}\left\langle n_{0}^{\mathrm{d}}\right\rangle+V^{2}\right)} .
$$

Introducing the above result in equation (11) the $f$ f Green functionwhich exhibits a tri-modal structure for the $4 \mathrm{f}$-spectral density of states, associated to the higher order of the approximaton on the self-energy $\gamma^{\mathrm{ff}}(w)$.

If one goes further in our perturbative treatment one can obtain, in principle, a $n$-modal structure for the f-f propagator. However, for the physical situation which we are interested in, one needs only to go up to second order in $U$, where the main features of the 4 f-states structures are already present (cf. Eq. (19)).

Finally, it should be mentioned, that this approach can also be applied in the case of strong correlation limit, i.e., $U / \Delta \gg 1$. In this case, the choice of the starting set of operators is a different one, namely:

$$
\begin{aligned}
\left\langle A_{i}^{+}\right\rangle & =\left\{f_{0 \sigma} n_{0}^{\mathrm{d}+}, d_{\mathbf{k}_{\sigma}}\right\} \\
\left\langle A_{i}^{-}\right\rangle & =\left\{f_{0 \sigma} n_{0}^{\mathrm{d}-}, d_{\mathbf{k}_{\sigma}}\right\} \\
\left\langle B_{i}\right\rangle & =\left\{f_{o \sigma}, d_{\mathbf{k}_{\sigma}}\right\}
\end{aligned}
$$

where:

$$
\begin{aligned}
& n_{0}^{\mathrm{d}+}=n_{0}^{\mathrm{d}}, \\
& n_{0}^{\mathrm{d}^{-}}=1-n_{0}^{\mathrm{d}} .
\end{aligned}
$$

With this choice, the f-f propagator can be written as:

$$
{ }_{00 \sigma}^{\mathrm{ff}}(w)=G_{00 \sigma}^{\mathrm{ff}+}(w)+G_{00 \sigma}^{\mathrm{ff}-}(w)
$$

where

$$
G_{00 \sigma}^{\mathrm{ff} \pm}(w)=i \theta(t)\left\langle\left[f_{0 \sigma} n_{0}^{d \pm}, f_{0 \sigma}^{+}\right]\right\rangle
$$

In the lowest approximation and assuming $V=0$ (i.e., a Falicov-Kimball model), one gets the usual Hubbard-type bimodal structure

$$
G_{00 \sigma}^{\mathrm{ff}}(w)=\frac{1-\left\langle n_{0}^{\mathrm{d}}\right\rangle}{w-\varepsilon_{0}}+\frac{\left\langle n_{0}^{\mathrm{d}}\right\rangle}{w-\varepsilon_{0}-U},
$$

which is completely different from the bimodal structure derived in this work, in the weak correlation regime.

[1] Parks, R. D., Raaen, S., den Boer, M. L., Chang, Y. S. and Williams, G. P., Phys. Rev. Lett. 52, (1984) 2176.

[2] Wieliczka, D. M., Olson, C. G. and Lynch, D. M., Phys. Rev. Lett. 52 (1984) 2180.

[3] Gunnarson, O. and Schönhamer, K., Phys. Rev. Lett. 50 (1983) 604.

[4] Liu, S. H. and Ho, K. M., Phys. Rev. B 28 (1983) 4220.

[5] Riseborough, P. S., Physica 130B (1985) 66.

[6] Nunez-Requeiro, M. D. and Avignon, M., Phys. Rev. Lett. 55 (1985) 615.

[7] Fedro, A. J. and Wilson, R. S., Phys. Rev. B 11 (1975) 2148.

[8] Kishore, R., Phys. Rev. B 19 (1979) 3822.

[9] Chao, K. A., Kishore, R. and da Cunha Lima, I. C., J. Phys. C 11 (1978) L953.

[10] Troper, A. and da Cunha Lima, I. C., Solid State Commun. 61 (1987) 195.
NON LOCA

J. V. de

Instituto

Abstract. - A I keeping the term Lacroix and Cyro the nearest neigh

Introduction

Many attem ground state 0 such a system the RKKY in netic phase, a pensate the $\mathrm{m}$ for the inexist $\mathrm{CeAl}_{3}$ or the $\mathrm{e}$ earth compour On the other $\mathrm{b}$ Wolff transforr the Anderson tice one. In $t$. W transforma Hamiltonian, cal Kondo inte nearest neighb this term on $t$ $T=0 \mathrm{~K}$.

The model a

A detailed Here we preser the case of on

$$
\begin{array}{r}
H \\
H_{0}=\sum_{k} \\
H_{1}= \\
H_{2}= \\
H_{3}=-J_{1} \sum_{i, j, \sigma}
\end{array}
$$

